Case Report

\title{
Emergence of GI.6 Outbreaks in a High School in Fangshan District, Beijing, China
}

\author{
Liu Haibo*, Shi Wengfeng \\ Microbiology Laboratory, Fangshan District Center for Disease Control and Prevention, Beijing, China \\ Email address: \\ liuhaibo0917@126.com (Liu Haibo),swf0603@163.com (Shi Wengfeng) \\ *Corresponding author
}

\section{To cite this article:}

Liu Haibo, Shi Wengfeng. Emergence of GI.6 Outbreaks in a High School in Fangshan District, Beijing, China. Science Journal of Public Health. Special Issue: Academic Research for Multidisciplinary. Vol. 6, No. 4, 2018, pp. 106-110. doi: 10.11648/j.sjph.20180604.11

Received: June 24, 2018; Accepted: July 7, 2018; Published: July 30, 2018

\begin{abstract}
Background] An outbreak of gastroenteritis happened in a high school in Fangshan District, Beijing, China in March 2016, in which 44 students developed the symptoms of vomiting, nausea, diarrhea and abdominal pain. Epidemiological investigation, laboratory investigation and Statistical analysis were conducted to identify the agent and source of this outbreak.[Methods] A case-control study was performed to discover the factors associated with this infection. Questionnaires and clinical data from those infection-exposed people were assessed. Here an outbreak case was defined as a student who developed at least three following symptoms: diarrhea, vomiting, abdominal pain and nausea in 72 hours after the lunch on 2 nd March., and controls were those who study or work in the school but did not have lunch on the day. Samples (Stool samples, rectal swabs and vomit samples) from infection-exposed students and controls, as well as environmental samples like food were collected to test for the existence of any suspicious bacteria or viruses. Detection of norovirus wasdone by real-time TaqMan RT-PCR and sequence analysis. Chi-square test was used to decide whether those differences could be of any statistical significance. [Results] The total number of respondents with complete data was 44 . An epidemiological data combined with the epidemic curve indicated that the outbreak started initially from a point source type, and was followed by a secondary transmission. Five stool samples, three rectal swabs and one vomit sample from case group turned out to be positive for norovirus genotype I by real-time PCR, and two asymptomatic food handlers in control group were positive for NoV GI. Sequence analysis of GI positive sample confirmed that the norovirus GI.6 variant was the etiological agent of the outbreak. Comparasion between the tested results from samples of those students who had lunch in school canteen and those who did notindicates that the difference of incidence was of statistical significance $(\mathrm{P}<0.01,95 \%$ CI:4.22-324.41). Food and environmental samples were tested to be bacteria-negative. [Conclusions] Our result suggested that the etiological agent of the outbreak was norvirus GI.6, which leads to gastroenteritis. We identified that asymptomatic infected food handlers were most likely to be the source of the outbreak. Therefore, hand hygiene practices strict adherence to regulations and access to hand washing facilities should be strengthened.
\end{abstract}

Keywords: Norovirus, School, Food Handler, Outbreak

\section{Introduction}

Noroviruses are divided into 5 genogroups (GI-GV) of which GI, GII and GIV could cause gastroenteritis in humans, and they are the major cause of gastroenteritis among all age groups worldwide [1-3], including foodborne outbreaks, and a major cause of sporadic gastroenteritis [4-6]. Norovirus is so highly infectious that it can be transmitted in various ways including person to person [7], contaminated environment or airborne contamination [8] and food [6]. It is reported that the food worker related factors contribute 70 percent of the source of the food contamination in the foodborne norovirus outbreak [9]. As has been widely reported, the dominant cause to the increasing number of norovirus outbreaks was genotype GII. 4 virus, while the GI noroviruses seems to be a relatively uncommon one, whose outbreak epidemiology and 
characteristics are scarce $[10,11]$. In China, norovirus infections were frequently reported, and GII is the common genogroup $[12,13]$. In the paper, for the first time, we will report the emergency of GI.6 nonrovirus as a cause of outbreaks in Fangshan District, Beijing, China and discuss its effect on public health.

In the Emerence of GI.6 outbreak happened in the high school located in the Fangshan District, Beijing, China, seven gastroenteritis cases among students having lunch in the school carteen were found in the high school on $3^{\text {rd }}$ March 2016 by a local hospital. Investigation was conducted by Fangshan District Center for Disease Control and Prevention of Beijing to pindown the etiological agent. To detect the existence of norovirus and further quantify the target genes in the samples, the real-time PCR is adopted for it is much more sensitive and specific than conventional PCR.

\section{Materials and Methods}

\subsection{Epidemiological Investigation}

An epidemiological investigation was conducted to spot the possible causes. We define a suspected case for a student in this high school with at least three of the following symptoms: vomiting, nausea, diarrhea and abdominal pain, Confirmed cases for those Suspected cases with positive laboratory confirmation of exsistence of the norovirus, and Controls for students or staffs without symptoms of diarrhea and vomiting within the longest incubation period of norovirus infection (72 h) after exposure to suspected risk factors. Demographicclinical data and Questionnaire survey were conducted to investigate the conditions of food and water that the students used. Survey and tested Data were analyzed by Excel, version 2010(Microsoft) and SPSS for Windows, version 18.0 (SPSS Inc, USA). Categorical variables for exposure of students and controls were analyzed by Chi-square or Fisher's exact test. All comparisons were two tailed and $P$-value $<0.05$ was considered significant.

\subsection{Samples Collection}

The high school students involved in the study were below 18 years of age, we had written informed consent from the human subjects. Their guardians have signed informed consent. We have requested permission before we got the student's rectal swab. We tried our best to collect vomit samples of the cases, the stool stample if the vomit samples were not available, and the rectal swab sample if the stool stemples are no available. Finally, a total of 24 clinical samples (including 2 vomit, 7 stool and 15 rectal swab samples) were collected from case group, then we collected 20 clinical samples from control group and 10 from school canteen food handlers, staffs and teachers (including 3 stool and 7 rectal swab samples), 10 from students without infection exposure (including10 rectal swab samples). Meanwhile, every food item was aseptically collected from canteen. For the purpose of detecting highly transmissible environmental contagious agents, samples like surface swabs from canteen, classroom tables, kitchen rags, kitchen cabinet, the knobs of the classroom, communal water cooler spigot in the affected classroom and direct drinking water were collected. As soon as the collection was finished, clinical samples and food samples were immediately sent to the laboratory for testing.

\subsection{Laboratory Testing}

All clinical samples were sent to the Fangshan District Center for Disease Control and Prevention (CDC) of Beijing for bacterial and viral tests, and sequencing and sequence analysis were also performed by the Beijing CDC. Bacterial tests included isolation, culture and purification for Staphylococcus aureus, Bacillus cereus, Salmonella spp. and diarrhoeagenic Escherichia coli. After RNA extraction were done by Roche MagNA Pure LC instrument (Roche Applied Science, IN, USA), the vomits, stools, rectal swabs, food samples and concentrated water sample were tested for existence of viral agent by real-time TaqMan RT-PCR on Roche LightCycler 480 using corresponding detection kit (Jiangsu Shuoshi Bio-TechCo, Ltd, Jiangsu, China).

PCR products were purified and sequenced directly on an ABI $3730 \times 1$ DNA Analyzer using a BigDye Terminator v3.1 Cycle Sequencing Kit (ABI, Austin, TX, USA). All sequences were prepared and aligned by BioEdit (version 7.0.9.0) with the Clustal W program. Genotypes were determined by phylogenetic analyses with the Norovirus Typing Tool (available at http://www.rivm.nl/mpf/norovirus/typingtool). The phylogenetic tree was constructed using the maximum likelihood method with MEGA software (version 6.06) and bootstrap analysis was performed with 1000 replications.

\subsection{Ethical Statement}

This study was approved by the Ethics Committee of the Fangshan District Center for Disease Control and Prevention of Beijing.

\section{Results}

\subsection{Epidemiological Investigation}

The first case was recognized on 3rd March 2016. Up to 5th March 2016, a total number of 38 cases were identified. Figure 1 shows the distribution of cases by date of symptom onset. The main symptoms were vomiting (94.7\%), diarrhea (57.9\%), nausea $(57.9 \%)$, abdominal cramp $(55.26 \%)$ and fever $(31.6 \%)$. 


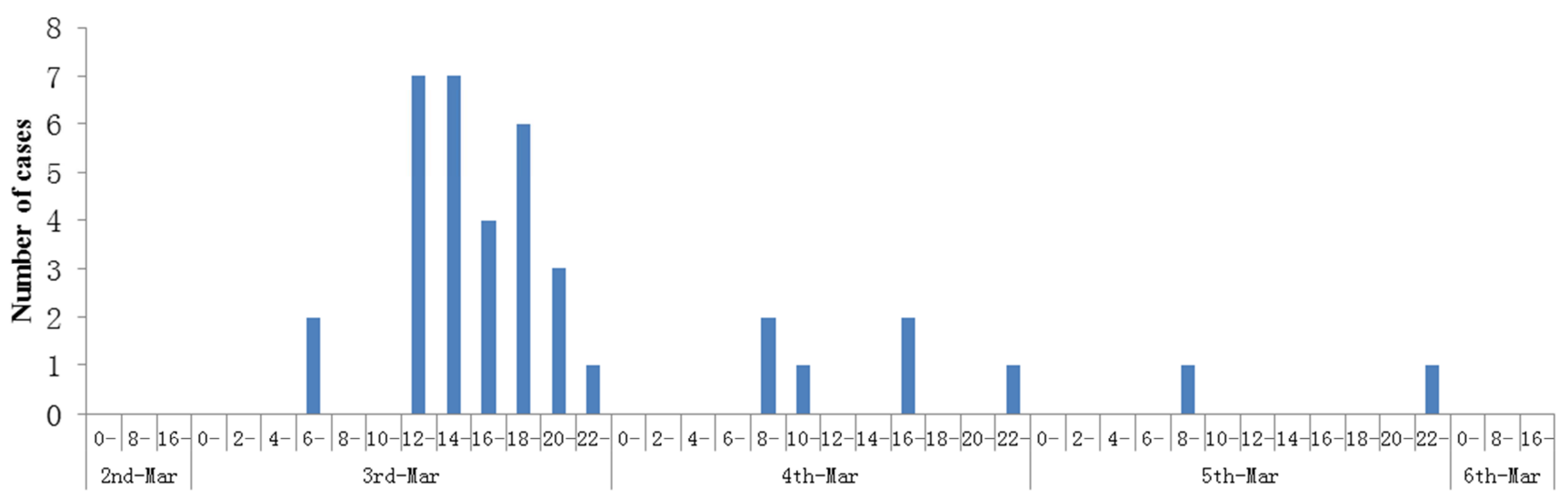

\section{Date and full hour of symptom onset}

Figure 1. Cases of outbreak of norovirus gastroenteritis in the high school.

Risk factor analysis, as shown in Table 1, showed that the incidence of gastroenteritis among those who had lunch in school canteen were 37.0-fold (95\%CI:4.22-324.41), higher than those who did not, and the incidence of gastroenteritis among those who live in school were 35.0-fold (95\%CI:7.40-165.55), higher than those who did not live in school $(\mathrm{P}<0.05)$.

Table 1. Risk factors analysis table.

\begin{tabular}{lllll}
\hline Variables & Case $\boldsymbol{n}(\mathbf{\%})$ & Control $\boldsymbol{n}(\mathbf{\%})$ & $\boldsymbol{P}$-valued & OR (95\%CI) \\
\hline Have lunch in school canteen & 38 & 20 & & \\
Yes & 37 & 10 & $<0.01$ & $37.0(4.22-324.41)$ \\
No & 1 & 10 & & \\
Live in school & 38 & 20 & $<0.01$ & $35.0(7.40-165.55)$ \\
Yes & 35 & 5 & & \\
No & 3 & 15 & & \\
\hline
\end{tabular}

\subsection{Detection Results of Gastroenteritis Viruses}

Norovirus was not detected in surface swabs from canteen, classroom tables, kitchen rags, kitchen cabinet, the knobs of the classroom, communal water cooler spigot, drinking water and food samples. Nevertheless, norovirus was found positive in 9 clinical samples $(37.5 \%, 9 / 24$; including 1 vomit, 5 stool and 3 rectal swab samples) in case group. Norovirus was tested to be positive in 2 clinical samples $(10 \%, 2 / 20$; including 2 rectal swab samples) in control group, and the positive samples belong to the food handlers. GI.6 was the genotype. Table 2 shows the results of sample collection and detection.

Table 2. Results of samples collection and detection.

\begin{tabular}{|c|c|c|c|c|c|}
\hline \multirow{2}{*}{ Tape of samples } & \multirow{2}{*}{ Numbers } & \multirow{2}{*}{ Bacterial pathogens* } & \multicolumn{3}{|c|}{ Norovirus } \\
\hline & & & Positive & Negative & Genotype \\
\hline Vomitus, stool and rectal swab & 44 & 0 & 11 & 33 & GI.6 \\
\hline Food & 15 & 0 & 0 & 15 & NT \\
\hline Highly transmissible environmental contagious agents & 50 & 0 & 0 & 50 & NT \\
\hline Direct drinking water & 51 & 0 & 0 & 51 & NT \\
\hline
\end{tabular}

*Detected bacterial pathogens include Staphylococcus aureus, Bacillus cereus, Salmonella spp. and diarrhoeagenic Escherichia coli.

NT:samples were not tested.

\section{Discussion}

GII.4 has been the majority of clinical case [14] as previous epidemiologic data suggest, and GI.6 cases were reported in United States [15], Denmark [16] and Australia [17] etc. While in China, it is reported that the GII is the common genogroup in the norovirus infections issue [18-20], and the noroviruses is associated with sporadic gastroenteritis.

The outbreak of acute gastroenteritis, reported on 3rd
March 2016 in a high school in Fangshan District, Beijing, China, was to be caused by GI. 6 because most of the affected students became symptomatic within 24-48 hours after having lunch on 2nd March 2016, accompanied with the predominant clinical symptoms of vomiting, abdominal pain and diarrhea, characteristic of norovirus infection. Norovirus is the most frequently reported cause of gastroenteritis outbreaks, which is transmitted through person-to-person contact, the most common mode of transmission [21, 22]. The epidemic curve 
of the outbreak suggested a common point source, and the case-control study identified that having lunch in school canteen was a highly risk factor. Since norovirus can survive at a temperature as high as 60 degrees, consumption was made that incompletely-cooked or ready-to-eat food with norovirus contamination would be high risk of infection [23]. Foodborne transmission is a significant way of spreading noroviruses globally, and it happens either when food handlers contaminated the food on site or during the earlier steps of food production [24, 25]. Compared to GII strains 7, Genogroup GI strains are more likely to be present in waterborne transmission, because GI strains have a higher stability in water than GII strains [26]. But, in the outbreak, noroviruses were not detected in the water samples.

According to the analyses of risk factors, students having lunch in school canteen and living in school increasthe risk of the transmission of the norovirus. Negative tested results for norovirus in drinking water and food samples and highly transmissible environmental contagious agents did not support the hypothesis of food-borne, water-borne or environmental contamination origin of the outbreak. The results of investigation of this outbreak of gastroenteritis suggested that the source of the outbreak were likely to be the two asymptomatic food handlers who were norovirus GI.6 tested positive. Asymptomatic food handlers were found highly contaminated by norovirus on a test on norovirus-associated gastroenteritis in food-catering settings [27]. Meanwhile, the existence of norovirus RNA were detected on the samples from the hands of food handlers, which would illustrate that the virus could be disseminated by virus-shedding food handlers [28]. Symptomatic food handlers were not allowed to work with food until 48 hours after clear of norovirus symptoms. In practice hygiene practices such as proper hand washing and wearing gloves must be strictly carried out according to the regulations while preparing foods in order to prevent the occurring and spreading of norovirus.

\section{Conclusions}

The culprit of the gastroenteritis outbreak happening in Fangshan District, Beijing was detected to be norovirus GI.6 which is a foodborne norovirus, and the food handlers were traced back to as the source of this norovirus from the food supply chain. Therefore, hygiene practices according to the regulations, such as thorough hand hygiene and use of gloves and protective clothing in kitchen areas, must be strictly followed during the process of food supply to prevent the occurring and spreading of norovirus.

\section{References}

[1] E Robilotti, S Deresinski, BA Pinsky. Norovirus. Clin Microbiol Rev. 2015; 28(1):134-64.

[2] AJ Hall, BA Lopman, DC Payne, et al. Norovirus disease in the United States. Emerg Infect Dis. 2013; 19(8):1198-205.
[3] SM Ahmed, AJ Hall, AE Robinson, et al. Global prevalence of norovirus in cases of gastroenteritis: a systematic review and meta-analysis. Lancet Infect Dis. 2014; 14(8):725-30.

[4] AJ Hall, ME Wikswo, $\mathrm{K}$ Manikonda, et al. Acute gastroenteritis surveillance through the National Outbreak Reporting System, United States. Emerg Infect Dis. 2013; 19(8):1305-9.

[5] J Mans, GE Armah, AD Steele, et al. Norovirus Epidemiology in Africa: A Review. PLoS One. 2016; 11(4):e0146280.

[6] MY Chen, WC Chen, PC Chen, et al. An outbreak of norovirus gastroenteritis associated with asymptomatic food handlers in Kinmen, Taiwan. BMC Public Health. 2016; 16372.

[7] ME Wikswo, A Kambhampati, K Shioda, et al. Outbreaks of Acute Gastroenteritis Transmitted by Person-to-Person Contact, Environmental Contamination, and Unknown Modes of Transmission--United States, 2009-2013. MMWR Surveill Summ. 2015; 64 (12): 1-16.

[8] S Xiao, JW Tang, Y Li. Airborne or Fomite Transmission for Norovirus? A Case Study Revisited. Int J Environ Res Public Health. 2017; 14 (12): E1571.

[9] AJ Hall, ME Wikswo, K Pringle, et al. Vital signs: foodborne norovirus outbreaks - United States, 2009-2012. MMWR Morb Mortal Wkly Rep. 2014; 63(22):491-5.

[10] KC Smith, T Inns, V Decraene, et al. An outbreak of norovirus GI-6 infection following a wedding in North West England. Epidemiol Infect. 2017; 145 (6):1239-1245.

[11] LD Bruggink, O Oluwatoyin, R Sameer, et al. Molecular and epidemiological features of gastroenteritis outbreaks involving genogroup I norovirus in Victoria, Australia, 2002-2010. J Med Virol. 2012; 84(9):1437-48.

[12] SW Qin, TC Chan, J Cai, et al. Genotypic and Epidemiological Trends of Acute Gastroenteritis Associated with Noroviruses in China from 2006 to 2016. Int J Environ Res Public Health. 2017; 14(11): E1341.

[13] H Chen, S Wang, W Wang. Complete Genome Sequence of a Human Norovirus Strain from the United States Classified as Genotype GII. P6_GII. 6. Genome Announc. 2018; 6 (22): e00489-18.

[14] J Vinje. Advances in laboratory methods for detection and typing of norovirus. J Clin Microbiol. 2015; 53(2):373-81.

[15] E Leshem, L Barclay, M Wikswo, et al. Genotype GI. 6 norovirus, United States, 2010-2012. Emerg Infect Dis. 2013; 19(8):1317-20.

[16] L Muller, AC Schultz, J Fonager, et al. Separate norovirus outbreaks linked to one source of imported frozen raspberries by molecular analysis, Denmark, 2010-2011. Epidemiol Infect. 2015; 143(11):2299-307.

[17] LD Bruggink, NL Dunbar, MG Catton, et al. Norovirus genotype diversity associated with gastroenteritis outbreaks in Victoria in 2013. Commun Dis Intell Q Rep. 2015; 39(1):E34-41.

[18] QM Zheng, HT Zeng, CW Dai, et al. Epidemiological investigation of a norovirus GII. 4 Sydney outbreak in a China elder care facility. Jpn J Infect Dis. 2015; 68(1):70-4. 
[19] BJ Jones, J Flint, J Collins, et al. Gastroenteritis outbreak at a health function caused by an emerging recombinant strain of Norovirus GII. P16/GII. 4 Sydney 2012, Australia. Epidemiol Infect. 2018; 146 (8):970-971.

[20] MC Medici, F Tummolo, V Martella, et al. Emergence of novel recombinant GII. P16 GII. 2 and GII. P16 GII. 4 Sydney 2012 norovirus strains in Italy, winter 2016/2017. New Microbiol. 2018; 41 (1):71-72.

[21] R Solano, M Alseda, P Godoy, et al. Person-to-person transmission of norovirus resulting in an outbreak of acute gastroenteritis at a summer camp. Eur J Gastroenterol Hepatol. 2014; 26(10):1160-6.

[22] ME Wikswo, A Kambhampati, K Shioda, et al. Outbreaks of Acute Gastroenteritis Transmitted by Person-to-Person Contact, Environmental Contamination, and Unknown Modes of Transmission--United States, 2009-2013. MMWR Surveill Summ. 2015; 64 (12):1-16.

[23] Terio V, Bottaro M, Pavoni E, et al. Occurrence of hepatitis A and $\mathrm{E}$ and norovirus GI and GII in ready-to-eat vegetables in Italy. Int J Food Microbiol. 2017; 249: 61-65.
[24] L Verhoef, J Hewitt, L Barclay, et al. Norovirus genotype profiles associated with foodborne transmission, 1999-2012. Emerg Infect Dis. 2015; 21(4):592-9.

[25] C Rumble, S Addiman, S Balasegaram, et al. Role of Food Handlers in Norovirus Outbreaks in London and South East England, 2013 to 2015. J Food Prot. 2017; 80 (2):257-264.

[26] M de Graaf, J van Beek, MP Koopmans. Human norovirus transmission and evolution in a changing world. Nat Rev Microbiol. 2016; 14 (7): 421-33.

[27] HS Koo, MO Lee, PT Ku. Molecular epidemiology of norovirus in asymptomatic food handlers in Busan, Korea, and emergence of genotype GII. 17. J Microbiol. 2016; 54 (10): 686-94.

[28] MY Chen, WC Chen, PC Chen, et al. An outbreak of norovirus gastroenteritis associated with asymptomatic food handlers in Kinmen, Taiwan. BMC Public Health. 2016; 16: 372. 